G. Tamone

Nagoya Math. J.

Vol. 94 (1984), 75-87

\title{
A CONNECTION BETWEEN BLOWING-UP AND GLUINGS IN ONE-DIMENSIONAL RINGS
}

\author{
GRAZIA TAMONE
}

\section{Introduction}

Let $C$ be an affine curve, contained on a non-singular surface $X$ as a closed 1-dimensional subscheme. If $P$ is a closed point on $C$, the blowing-up $C^{\prime}$ of $C$ with center $P$ (induced by the blowing-up of $X$ with center $P$ ) is an affine curve. It is known that there is a sequence:

$$
\bar{C}=C_{k} \longrightarrow C_{k-1} \longrightarrow \cdots \longrightarrow C_{1} \longrightarrow C_{0}=C,
$$

where $\bar{C}$ is the normalization of $C$, and each $C_{i+1}$ is the blowing-up of $C_{i}$ with center a singular point $P_{i}$ on $C_{i}(i=0, \cdots, k-1)$.

The sequence $(\cdot)$ induces a sequence of rings:

$$
R=R_{0} \subset R_{1} \subset \cdots \subset R_{k-1} \subset R_{k}=\bar{R},
$$

where, for $j=0, \cdots, k, R_{j}$ is the coordinate ring of $C_{j}$; for each $i=$ $0, \cdots, k-1, R_{i+1}$ is called the ring "obtained from $R_{i}$ by blowing-up the maximal ideal of $R_{i}$ corresponding to $P_{i}$ ”.

On the other hand, there is also a sequence between $R$ and $\bar{R}$ :

$$
R=B_{n} \subset B_{n-1} \subset \cdots \subset B_{1} \subset B_{0}=\bar{R},
$$

where each $B_{i+1}(\mathrm{i}=0, \cdots, n-1)$ is a "gluing of primary ideals of $B_{i}$ over a prime ideal of $R$ " (see [6]).

In this paper we wonder under what assumptions a sequence $(*)$ is also a sequence $(* *)$ of gluings between $R$ and $\bar{R}$; in this case, the method of "gluing" defined in [6] is "inverse" of the process of "blowing-up" used to obtain the desingularization of $C$. We give necessary and sufficient conditions on $(*)$ in order that $(*)$ is also a sequence of gluings like $(* *)$; then, we show some classes of rings satisfying the required condition, in particular the rings considered in the last theorem of [7].

Received October 5, 1982.

This work was supported by C.N.R. (Consiglio Nazionale delle Ricerche). 
$\S 1$.

Let $C$ be an affine curve, $P_{1}, \cdots, P_{n}$ the singular points on $C, R$ the coordinate ring of $C$. For $i=1, \cdots, n$, the maximal ideal of $R$ corresponding to $P_{i}$ is a prime ideal belonging to the conductor $\mathfrak{b}$ of $R$ in $\bar{R}$. Then, if $\operatorname{Ass}(R / \mathfrak{b})=\left\{\mathfrak{m}_{1}, \cdots, \mathfrak{m}_{n}\right\}$, and $S=R-\cup \mathfrak{m}_{i}$, the ring $A=S^{-1} R$ is semilocal, and its maximal ideals are exactly $\mathrm{m}_{1} A, \cdots, \mathfrak{m}_{n} A$, so that the maximal ideals of $A$ correspond to the singular points of $C$. Besides, if $R^{\prime}$ is the coordinate ring of the blowing-up of $C$ with center $P_{i}$ $(i=1, \cdots, n)$, the ring "obtained from $A$ by blowing-up $\mathfrak{m}_{i} A$ " is canonically isomorphic to $S^{-1} R^{\prime}$ ([4], p. 663). Owing to these facts, we can consider $A$ instead of $R$ without loss of generality.

Since $A$ is semilocal, the ring "obtained from $A$ by blowing-up a maximal ideal $\mathrm{m}$ " can be described in various ways, according to [4] and [5]. In fact, if $A$ is a semilocal 1-dimensional Cohen-Macaulay ring, the ring obtained by blowing-up $\mathfrak{m} \in \operatorname{Spm}(A)$ coincides with the "first neighbourhood of $A$ ": $\Lambda=\left\{b / a \mid b \in \mathfrak{m}^{s}, a\right.$ is superficial of degree $\left.s\right\}$, defined in [5], Chapter XII. This ring can also be written as $A\left[z_{1} / x, \cdots, z_{t} / x\right]$, where $\left\{z_{1}, \cdots, z_{t}\right\}$ is a set of generators of $\mathfrak{m}, x \in \mathfrak{m}$ is $\mathfrak{m}$-transversal; besides, this ring coincides with $\mathfrak{m}^{n}: \mathfrak{m}^{n}=\left\{a \in \bar{A} \mid a \mathfrak{m}^{n} \subset \mathfrak{m}^{n}\right\}$ for all sufficiently large $n$ (see [4], Proposition 1.1, Definition 1.7, Lemma 1.8, and [2], Corollary 3.5).

In this paper, unless we give further notice, $A$ will mean a semilocal 1-dimensional Cohen-Macaulay ring. Besides, we shall denote the "embedding dimension" and the "multiplicity" of a local ring $S$ respectively by: emdim $(S)$ and $e(S)$.

First of all, we prove some lemmas we need to study some conductors which we are interested in.

Lemma 1.1. Let $\mathfrak{p}$ be a maximal ideal in $A, A$ be the ring obtained from $A$ by blowing-up $\mathfrak{p}$. If $A \neq \Lambda$, the conductor of $A$ in $\Lambda$ is a p-primary ideal.

Proof. Let $\mathfrak{a}$ be the conductor of $A$ in $\Lambda$. As seen before, $\Lambda=\mathfrak{p}^{n}: \mathfrak{p}^{n}$ for a suitable $n$, so, for each $x \in \mathfrak{p}, y \in \Lambda$ we have: $y x^{n} \in \mathfrak{p}^{n}$, thence $x^{n} \Lambda \subset A$. It follows: $x^{n} \in \mathfrak{a}$ for each $x \in \mathfrak{p}$, so $\mathfrak{p} \subset \sqrt{\bar{a}}$. Now, $\mathfrak{p}$ is maximal and $\mathfrak{a}$ is a proper ideal, then we have $\mathfrak{p}=\sqrt{ } \overline{\mathfrak{a}}$ and $\mathfrak{a}$ is $\mathfrak{p}$-primary.

Corollary 1.2. Let $\mathfrak{b}$ be the conductor of $A$ in $\bar{A}, \Lambda$ be the ring obtained from $A$ by blowing-up a maximal ideal $\mathfrak{p}$ belonging to $\mathfrak{b}$. If $\mathfrak{p}$ coincides with the $\mathfrak{p}$-primary component of $\mathfrak{b}$, the conductor of $A$ in $\Lambda$ is $\mathfrak{p}$. 
Proof. We first have: $A \neq \Lambda$ : in fact, $A=\Lambda$ implies $\mathfrak{p}=\mathfrak{p} \Lambda=x \Lambda=$ $x A$ for some regular element $x \in A$ ([4], Proposition 1.1, (ii)), so $A_{\mathfrak{p}}$ is regular, then $A_{\mathfrak{v}}=\overline{A_{\mathfrak{p}}}$ while $\mathfrak{p} \in \operatorname{Ass}(A / \mathfrak{b})$. So, $A \subsetneq A \subset \bar{A}$; then, if $\mathfrak{a}$ is the conductor of $A$ in $\Lambda$, we have $\mathfrak{b} \subset \mathfrak{a}$, and also $\sqrt{\mathfrak{a}}=\mathfrak{p}$ (Lemma 1.1). Let $\mathfrak{q}$ be the $\mathfrak{p}$-primary ideal belonging to $\mathfrak{b}$; the reduced primary decomposition of $\mathfrak{b}$ is like this: $\mathfrak{b}=\mathfrak{q} \cap\left(\cap \mathfrak{q}_{j}\right)$. Then, if $\mathfrak{p}=\mathfrak{q}$, owing to the above facts we have: $\mathfrak{p} \cap\left(\cap \mathfrak{q}_{j}\right) \supset \mathfrak{a} \cap\left(\cap \mathfrak{q}_{j}\right) \supset \mathfrak{b}=\mathfrak{q} \cap\left(\cap \mathfrak{q}_{j}\right)=\mathfrak{p} \cap\left(\cap \mathfrak{q}_{j}\right)$, hence:

$$
\mathfrak{p} \cap\left(\cap \mathfrak{q}_{j}\right)=\mathfrak{a} \cap\left(\cap \mathfrak{q}_{j}\right) \text {, with } \sqrt{\overline{\mathfrak{a}}}=\mathfrak{p} .
$$

It follows that the two sides of $(\cdot)$ are two reduced primary decompositions of the same ideal $\mathfrak{b}$, whose primary components are all isolated; then, owing to the uniqueness of these components, we have, in particular, $\mathfrak{p}=\mathfrak{a}$.

Remarks. 1) In general, if $\mathfrak{p}$ doesn't coincide with the $\mathfrak{p}$-primary component of $\mathfrak{b}$, one has: $\mathfrak{p} \neq \mathfrak{a}$. As an example, let us consider the ring $A=k \llbracket t^{3}, t^{5} \rrbracket$. The conductor $\mathfrak{b}$ of $A$ in $\bar{A}=k \llbracket t \rrbracket$ is $p$-primary, where $\mathfrak{p}=\left(t^{3}, t^{5}\right)$. We have: $\Lambda=A\left[t^{3} / t^{3}, t^{5} / t^{3}\right]$ ([4], Definition 1.7, Lemma 1.8, and the beginning of Section 1$)=k \llbracket t^{2}, t^{3} \rrbracket$. Let $\mathfrak{a}$ be the conductor of $A$ in $A$. One ean easily show that $\mathfrak{a} \neq \mathfrak{p}$, seeing that $t^{5} \in \mathfrak{p}, t^{5} \notin \mathfrak{a}$ because $t^{5} t^{2}=t^{7} \notin A$.

2) The inverse of Corollary 1.2 is not true, i.e. in some cases the conductor of $A$ in $A$ is $\mathfrak{p}$, but $\mathfrak{p}$ is not a primary ideal belonging to $\mathfrak{b}$. For example, if $A=k \llbracket t^{2}, t^{5} \rrbracket$, we have: $\bar{A}=k \llbracket t \rrbracket, \quad \mathfrak{b}=\left(t^{4}, t^{5}\right)$ is $\left(t^{2}, t^{5}\right)$ primary, and $\mathfrak{b} \neq\left(t^{2}, t^{5}\right)$. One has: $A=A\left[t^{2} / t^{2}, t^{5} / t^{2}\right]$ ([4], Proposition 1.1, Definition 1.7, Lemma 1.8) $=k \llbracket t^{2}, t^{3} \rrbracket$. Now, we show the conductor a of $A$ in $\Lambda$ is $\left(t^{2}, t^{5}\right)$. Owing to the maximality of $\left(t^{2}, t^{5}\right)$ it is enough to prove: $\left(t^{2}, t^{5}\right) \subset$ a. So, for each $x \in\left(t^{2}, t^{5}\right)$, we must prove $x \Lambda \subset A$. Let $x \in\left(t^{2}, t^{5}\right)$, $y \in \Lambda$; then, $x=t^{2} \sum a_{i j} t^{2 i} t^{5 j}+t^{5} \sum b_{h k} t^{2 h} t^{5 k}, \quad y=\sum c_{p q} t^{2 p} t^{3 q}$. So, $x y=$ $\sum c_{p q} t^{2 p}\left(x t^{3 q}\right)$. Now, $x t^{3 q}=\left(\sum a_{i j} t^{2 i} t^{5 j}\right) t^{3 q+2}+\left(\sum b_{h k} t^{2 h} t^{5 k}\right) t^{3 q+5}=\sum a_{i j} t^{2 i+5 j+3 q+2}$ $+\sum b_{h k} t^{2 h+5 k+3 q+5}$, and we have: $2 i+5 j+3 q+2 \geqslant 4$, or $=2$ for $i, j, q \in N$, $2 h+5 k+3 q+5 \geqslant 7$, or $=5$ for $h, k, q \in N$. So, $x t^{3 q} \in A$. Then, $x y=$ $\sum c_{p q} t^{2 p}\left(x t^{3 q}\right) \in A$, since also $t^{2 p} \in A$ for each $p$.

Corollary 1.3. Let $\mathfrak{p}, \Lambda$ be as in Lemma 1.1. If $\mathfrak{p}^{\prime}$ is a prime ideal of $A$, and $\mathfrak{p}^{\prime} \neq \mathfrak{p}$, there is a unique prime in $A$ over $\mathfrak{p}^{\prime}$.

Proof. Owing to Lemma 1.1, the conductor a of $A$ in $\Lambda$ is such that $\sqrt{\mathfrak{a}}=\mathfrak{p}$; then, if $\mathfrak{p}^{\prime} \neq \mathfrak{p}$, one has $\mathfrak{p}^{\prime} \not \supset \mathfrak{a}$ (otherwise $\mathfrak{p}^{\prime} \supset \mathfrak{p}$, and this implies 
$\left.\mathfrak{p}^{\prime}=\mathfrak{p}\right)$. It follows: $A_{\mathfrak{p}^{\prime}}=\Lambda_{A-\mathfrak{p}^{\prime}}$, so there is a unique prime ideal in $\Lambda$ over $\mathfrak{p}^{\prime}$ (since there is one-to-one correspondence between $\left\{\mathfrak{P} \in \operatorname{Spec} \Lambda / \mathfrak{P} \cap A=\mathfrak{p}^{\prime}\right\}$ and $\operatorname{Spec}\left(\Lambda_{A-\mathfrak{p}^{\prime}} / \mathfrak{p}^{\prime} \Lambda_{A-\mathfrak{p}^{\prime}}\right)=\operatorname{Spec}\left(A_{\mathfrak{p}^{\prime}} / \mathfrak{p}^{\prime} A_{\mathfrak{p}^{\prime}}\right)=\operatorname{Spec}\left(k\left(\mathfrak{p}^{\prime}\right)\right)$.

The next lemma holds in the general case: so, the rings considered here are not necessarily of the above type.

Lemma 1.4. Let $A, B, C$ be rings such that $A \subset B \subset C$, and let $\mathfrak{a}, \mathfrak{b}, \mathfrak{b}^{\prime}$ be respectively the conductor of $A$ in $B$, of $A$ in $C$, of $B$ in $C$. Then, $\mathfrak{a} \mathfrak{b}^{\prime} \subset \mathfrak{b}$ in $B$.

Proof. For each $x \in \mathfrak{a}, y \in \mathfrak{b}^{\prime}, c \in C$ we have (in $\left.B\right):(x y) c=x(y c)$, where $y c \in B$, since $y \in \mathfrak{b}^{\prime}$; so, $x(y c) \in A$ because $x \in \mathfrak{a}$. Then, $(x y) c \in A$, so that $x y \in \mathfrak{b}$. It follows that $\mathfrak{a} \mathfrak{b}^{\prime} \subset \mathfrak{b}$.

LEMMA 1.5. Under the assumptions of Corollary 1.2 , let $\mathfrak{a}, \mathfrak{b}^{\prime}$ be respectively the conductor of $A$ in $\Lambda$ and of $\Lambda$ in $\bar{A}$. If $\mathfrak{p}_{i} \in \operatorname{Ass}(A / \mathfrak{b})-\{\mathfrak{p}\}$, and $S=A-\mathfrak{p}_{i}$ we have $\mathfrak{b} S^{-1} \Lambda=\mathfrak{b}^{\prime} S^{-1} \Lambda$.

Proof. We have $\mathfrak{b} \Lambda \subset \mathfrak{b}^{\prime}$, since $(\mathfrak{b} \Lambda) \bar{A} \subset \mathfrak{b} \bar{A} \subset A \subset \Lambda$, so $\mathfrak{b} S^{-1} \Lambda \subset \mathfrak{b}^{\prime} S^{-1} \Lambda$. On the other hand, in $\Lambda$ one has $\mathfrak{a} \mathfrak{b}^{\prime} \subset \mathfrak{b}$ (Lemma 1.4), so $\left(\mathfrak{a} S^{-1} \Lambda\right)\left(\mathfrak{b}^{\prime} S^{-1} \Lambda\right)$ $=\left(\mathfrak{a} \mathfrak{b}^{\prime}\right) S^{-1} \Lambda \subset \mathfrak{b} S^{-1} \Lambda$, hence $\mathfrak{b}^{\prime} S^{-1} \Lambda \subset \mathfrak{b} S^{-1} \Lambda$ because $a S^{-1} \Lambda=S^{-1} \Lambda$ owing to the assumptions and Lemma 1.1 .

Using the above results, we can prove some facts concerning the conductor of $\Lambda$ in $\bar{A}$. We assume that $\bar{A}$ is a finite $A$-module.

Proposition 1.6. Let $\mathfrak{b}$ be the conductor of $A$ in $\bar{A}$, and Ass $(A / \mathfrak{b})=$ $\left\{\mathfrak{p}_{1}, \cdots, \mathfrak{p}_{n}\right\}$. Let $\Lambda_{j}$ be the ring obtained from $A$ by blowing-up $\mathfrak{p}_{j}(1 \leqslant j \leqslant n)$, $\mathfrak{b}_{j}$ be the conductor of $\Lambda_{j}$ in $\bar{A}$. The following facts hold:

1) for each $i \in\{1, \cdots, \hat{j}, \cdots, n\}$ there is a unique prime ideal $\mathfrak{B}_{i}$ in $\Lambda_{j}$ over $\mathfrak{p}_{i}$, and $\left\{\mathfrak{\beta}_{1}, \cdots, \hat{j}, \cdots, \mathfrak{B}_{n}\right\} \in \operatorname{Ass}\left(\Lambda_{j} / \mathfrak{b}_{j}\right)$

2) for each prime ideal $\mathfrak{B}$ in $\Lambda_{j}$ such that $\mathfrak{B} \cap A \neq \mathfrak{p}_{i}(i=1, \cdots, n)$ we have: $\mathfrak{B} \notin \operatorname{Ass}\left(\Lambda_{j} / \mathfrak{G}_{j}\right)$.

Proof. 1) For each $i \in\{1, \cdots, \hat{j}, \cdots, n\}$ we have $\mathfrak{p}_{i} \neq \mathfrak{p}_{j}$, so (Corollary 1.3) there is a unique prime in $\Lambda_{j}$ over $\mathfrak{p}_{i}$, say $\mathfrak{P}_{i}$. For each $\mathfrak{P}_{i} \in$ $\left\{\mathfrak{P}_{1}, \cdots, \hat{j}, \cdots, \mathfrak{P}_{n}\right\}$ we have $\mathfrak{P}_{i} \cap A=\mathfrak{p}_{\imath} \supset \mathfrak{b}$, so $\mathfrak{P}_{i} \supset \mathfrak{b} \Lambda_{j}$, thence if $S=A-\mathfrak{p}_{i}$, the ideal $\mathfrak{P}_{i} S^{-1} \Lambda_{j}$ is proper, and contains $\mathfrak{b} S^{-1} \Lambda_{j}$. Now, owing to Lemma 1.5, $\mathfrak{b} S^{-1} \Lambda_{j}=\mathfrak{b}_{j} S^{-1} \Lambda_{j}$. Then, we have: $\mathfrak{P}_{i} S^{-1} \Lambda_{j} \supset \mathfrak{b}_{j} S^{-1} \Lambda_{j} ;$ this implies $\mathfrak{P}_{2} S^{-1} \Lambda_{j}$ is in Ass $\left(S^{-1} \Lambda_{j} / \mathfrak{b}_{j} S^{-1} \Lambda_{j}\right)$, hence $\mathfrak{P}_{i} \in \operatorname{Ass}\left(\Lambda_{j} / \mathfrak{b}_{j}\right)$.

2) Let $\mathfrak{\beta} \in \operatorname{Spec}\left(\Lambda_{j}\right)$ be such that $\mathfrak{p}=\mathfrak{\beta} \cap A \neq \mathfrak{p}_{i}$ for $i=1, \cdots, n$. Then, $\mathfrak{p} \not \supset \mathfrak{b}$, so $A_{\mathfrak{p}}=\bar{A}_{A-p}$; it follows: $A_{\mathfrak{v}} \subset\left(\Lambda_{j}\right)_{A-\mathfrak{p}} \subset \bar{A}_{A-\mathfrak{p}}=A_{\mathfrak{p}}$, so $\left(\Lambda_{j}\right)_{A-\mathfrak{p}}$ 
$=\bar{A}_{A-p}$. Hence, the conductor $\mathfrak{b}_{j}\left(\Lambda_{j}\right)_{A-p}$ is not a proper ideal, so $\mathfrak{\beta} \notin$ Ass $\left(\Lambda_{j} / \mathfrak{b}_{j}\right)$ (otherwise $\mathfrak{B}\left(\Lambda_{j}\right)_{A-p}$, which is a proper ideal, would contain $\left.\mathfrak{b}_{j}\left(\Lambda_{j}\right)_{A-\mathfrak{p}}=\left(\Lambda_{j}\right)_{A-\mathfrak{p}}\right)$.

Proposition 1.7. Under the assumptions of Proposition 1.6, if $p_{j}$ coincides with the $\mathfrak{p}_{j}$-primary ideal belonging to $\mathfrak{b}$, then:

$\left\{\mathfrak{P} \in \operatorname{Spec} \Lambda_{j} \mid \mathfrak{B} \cap A=\mathfrak{p}_{j}\right\} \not \subset \operatorname{Ass}\left(\Lambda_{j} / \mathfrak{b}_{j}\right)$, so

$\left\{\mathfrak{P} \in \operatorname{Spec} \Lambda_{j} \mid \mathfrak{B} \cap A=\mathfrak{p}_{j}\right\} \cap \operatorname{Ass}\left(\Lambda_{j} / \mathfrak{b}_{j}\right) \quad$ is empty .

Proof. Let $S=A-\mathfrak{p}_{j}$; then, $\overline{S^{-1} A}=S^{-1} \bar{A}$, and the ring obtained from $S^{-1} A$ by blowing-up $\mathfrak{p}_{j} S^{-1} A$ is canonically isomorphic to $S^{-1} \Lambda_{j}$ (see the beginning of Section 1 ). Since $\mathfrak{p}_{j}$ equals the $\mathfrak{p}_{j}$-primary component of $\mathfrak{b}$, the conductor of $A$ in $\Lambda_{j}$ is $\mathfrak{p}_{j}$ (Corollary 1.2), so $\mathfrak{p}_{j} S^{-1} \Lambda_{j} \subset S^{-1} A$, then $\mathfrak{p}_{j} S^{-1} \Lambda_{j}=\mathfrak{p}_{j} S^{-1} A$. It follows: $S^{-1} \Lambda_{j}=\left\{x \in S^{-1} \bar{A} \mid x \mathfrak{p}_{j} S^{-1} A \subset \mathfrak{p}_{j} S^{-1} A\right\}$ ([4], Proposition 1.1 (i), Definition 1.3); besides, the conductor of $S^{-1} A$ in $S^{-1} \bar{A}$ is $\mathfrak{p}_{j} S^{-1} A$. All this allows us to prove: $S^{-1} \Lambda_{j}=S^{-1} \bar{A}$. Indeed, for each $x \in S^{-1} \bar{A}$ we have: $x\left(p_{j} S^{-1} A\right) \subset \mathfrak{p}_{j} \bar{S}^{-1} A \subset S^{-1} A$, so $x\left(\mathfrak{p}_{j} S^{-1} A\right) \subset \mathfrak{p}_{j} S^{-1} \bar{A} \cap S^{-1} A$ $=\mathfrak{p}_{j} S^{-1} A$, then $x \in S^{-1} \Lambda_{j}$. Now, let $\mathfrak{B} \in \operatorname{Spec} \Lambda_{j}$ be such that $\mathfrak{R} \cap A=\mathfrak{p}_{j}$; if $\mathfrak{\beta} \in \operatorname{Ass}\left(\Lambda_{j} / \mathfrak{b}_{j}\right)$, we have $\Re S^{-1} \Lambda_{j} \in \operatorname{Ass}\left(S^{-1} \Lambda_{j} / \mathfrak{b}_{j} S^{-1} \Lambda_{j}\right)$, while $\Re S^{-1} \Lambda_{j}$ is a proper ideal, and $\mathfrak{b}_{j} S^{-1} \Lambda_{j}$ is not a proper ideal, since $S^{-1} \Lambda_{j}=S^{-1} \bar{A}$. So, the result follows.

Remark. There are examples of rings $A$ such that $\mathfrak{p}_{j}$ doesn't equal the $\mathfrak{p}_{j}$-primary component of $\mathfrak{b}$, and Ass $\left(\Lambda_{j} / \mathfrak{b}_{j}\right)$ contains a prime ideal $\mathfrak{P}$ such that $\mathfrak{B} \cap A=\mathfrak{p}_{j}$. The ring $A=k \llbracket t^{3}, t^{5} \rrbracket$ and the ideal $\mathfrak{p}_{j}=\left(t^{3}, t^{5}\right)$ considered in remark 1) after Corollary 1.2 are an example of that. In fact, $\Lambda_{j}=k \llbracket t^{2}, t^{3} \rrbracket$, and the conductor $\mathfrak{b}_{j}$ is $\mathfrak{B}=\left(t^{2}, t^{3}\right)$; it is easily seen that $\mathfrak{B} \cap A=\left(t^{3}, t^{5}\right)=\mathfrak{p}_{j}$.

From Proposition 1.6 and Proposition 1.7 it follows immediately:

Corollary 1.8. Under the assumptions of Proposition 1.6, if $\mathfrak{p}_{j}$ coincides with the $\mathfrak{p}_{j}$-primary component of $\mathfrak{b}$, then $\operatorname{Ass}\left(\Lambda_{j} / \mathfrak{b}_{j}\right)=\left\{\mathfrak{P}_{1}, \cdots, \hat{j}, \cdots, \mathfrak{P}_{n}\right\}$ where $\mathfrak{P}_{i}$ is the only prime ideal in $\Lambda_{j}$ over $\mathfrak{p}_{i}$, for $i=1, \cdots, \hat{j}, \cdots, n$.

The following proposition shows another connection between the properties of the conductors $\mathfrak{b}$ and $\mathfrak{b}_{j}$.

Proposition 1.9. Let $A, \mathfrak{p}_{j}, \Lambda_{j}$ be as in Proposition 1.6, and $\mathfrak{B}_{i}$ be the only prime ideal in $A_{\jmath}$ over $\mathfrak{p}_{i}$, for $i=1, \cdots, \hat{j}, \cdots, n$. If $\mathfrak{p}_{i}$ coincides with the $\mathfrak{p}_{i}$-primary component of $\mathfrak{b}$, then $\mathfrak{P}_{i}$ coincides with the $\mathfrak{P}_{i}$-primary ideal belonging to $\mathfrak{b}_{j}$. 
Proof. Let $S=A-\mathfrak{p}_{i}$, a be the conductor of $A$ in $\Lambda_{j}$. Since $\mathfrak{p}_{i} \neq \mathfrak{p}_{j}$, we have: $\mathfrak{p}_{i} \not \supset \mathfrak{a}$, because $\mathfrak{a}$ is $\mathfrak{p}_{j}$-primary (Lemma 1.1) and $\mathfrak{p}_{j}$ is maximal; so, $S^{-1} A=S^{-1} \Lambda_{j}$. Moreover, $\mathfrak{b} S^{-1} \Lambda_{j}=\mathfrak{b}_{j} S^{-1} \Lambda_{j}$, owing to Lemma 1.5. So, $\mathfrak{b}_{j} S^{-1} \Lambda_{j}=\mathfrak{b} S^{-1} \Lambda_{j}=\mathfrak{b} S^{-1} A$, and this last ideal coincides with $\mathfrak{p}_{i} S^{-1} A$ because of the assumptions on $\mathfrak{p}_{i}$. Now, if $\mathfrak{Q}_{i}$ is the $\mathfrak{P}_{i}$-primary component of $\mathfrak{b}_{j}$, we have: $\mathfrak{b}_{j} S^{-1} \Lambda_{j}=\mathfrak{Q}_{i} S^{-1} \Lambda_{j}$. Then, $\mathfrak{Q}_{i} S^{-1} \Lambda_{j}=\mathfrak{p}_{i} S^{-1} A$. It follows that $\mathfrak{Q}_{i} S^{-1} \Lambda_{j}$ is a prime ideal; so, it coincides with its own radical $\mathfrak{P}_{i} S^{-1} \Lambda_{j}$. Thence, $\mathfrak{Q}_{i}=\mathfrak{P}_{i}$, because $\mathfrak{Q}_{i}$ is $\mathfrak{P}_{i}$-primary.

From Corollary 1.8 and Proposition 1.9 we get the following

Corollary 1.10. Let $A, \Lambda_{j}$ be as in Proposition 1.6 and let $\mathfrak{P}_{i}$ be the only prime ideal in $\Lambda_{j}$ over $\mathfrak{p}_{i}$, for $i \in\{1, \cdots, \hat{j}, \cdots, n\}$. If $\mathfrak{b}=\sqrt[A]{\mathfrak{b}}=$ $\bigcap_{i=1}^{n} \mathfrak{p}_{i}$ then $\mathfrak{b}_{j}=\sqrt[1]{j} \sqrt{\mathfrak{b}_{j}}=\bigcap_{i \neq j} \mathfrak{B}_{i}$.

$\S 2$.

Now, let

$$
A=A_{0} \subset A_{1} \subset \cdots \subset A_{k-1} \subset A_{k}=\bar{A}
$$

be a sequence where each $A_{j+1}$ is the ring obtained from $A_{j}$ by blowingup a prime ideal $\mathfrak{P}_{j}$ in $A_{j}(j=0, \cdots, k-1)$. We want to find necessary and sufficient conditions in order that $(*)$ is also a sequence

$$
A=B_{n} \subset B_{n-1} \subset \cdots \subset B_{1} \subset B_{0}=\bar{A},
$$

where each $B_{j+1}$ is the gluing, over a prime ideal $\mathfrak{p}$ of $A$, of the primary ideals belonging to $\mathfrak{p} B_{j}(j=0, \cdots, n-1)$. Now, $A_{j}$ in $(*)$ is the gluing, over a prime ideal $\mathfrak{p} \in \operatorname{Spec} A$, of the primary ideals of $\mathfrak{p} A_{j+1}$, if and only if $A_{j}$ is the gluing, over $\mathfrak{\beta}_{j} \cap A$, of the primary ideals of $\left(\mathfrak{\beta}_{j} \cap A\right) A_{j+1}$. In fact, if $A_{j}$ is the gluing, over a prime $\mathfrak{p}^{\prime}$ of $A$, of the primary ideals of $\mathfrak{p}^{\prime} A_{j+1}$, we have: $A_{j}=A+\mathfrak{p}^{\prime} A_{j+1}$, and $\mathfrak{P}^{\prime}=\mathfrak{p}^{\prime} A_{j+1}$ is a maximal ideal (see [7], Lemma 1.2,1)); besides, $\mathfrak{P}^{\prime}$ is the conductor of $A_{j}$ in $A_{j+1}$ (since $\mathfrak{P}^{\prime} A_{j+1}=\mathfrak{p}^{\prime} A_{j+1} \subset A_{j}$, and $\mathfrak{P}^{\prime}$ is maximal). Now, since $A_{j+1}$ is obtained from $A_{j}$ by blowing-up $\mathfrak{P}_{j}$, the conductor $\mathfrak{a}$ of $A_{j}$ in $A_{j+1}$ is such that $\sqrt{\mathfrak{a}}=\mathfrak{P}_{j}$ (Lemma 1.1). Then, we have: $\mathfrak{a}=\mathfrak{P}^{\prime}, \sqrt{\mathfrak{P}^{\prime}}=\sqrt{\mathfrak{a}}=\mathfrak{P}_{j}$, so $\mathfrak{P}_{\text {j }}$ $=\mathfrak{P}^{\prime}$. It follows: $\mathfrak{p}^{\prime}=\mathfrak{P}^{\prime} \cap A=\mathfrak{P}_{i} \cap A$, so $A_{j}$ is the gluing, over $\mathfrak{P}_{j} \cap A$, of the primary ideals belonging to $\left(\mathfrak{P}_{j} \cap A\right) A_{j+1}$. On the contrary, if each $A_{j}$ is the gluing, over $\Re_{j} \cap A$, of the primary ideals belonging to $\left(\mathfrak{P}_{j} \cap A\right) A_{j+1}$, then obviously $(*)$ is a sequence like $(* *)$. So, our problem is to require conditions in order that each $A_{j}$ is the gluing, over $\mathfrak{p}=\mathfrak{P}_{j} \cap A$, 
of the primary ideals of $p A_{j+1}$. We note that the property we are interested in implies the following (weaker) one: for $j=0, \cdots, k-1, A_{j}$ is the gluing, over $\mathfrak{B}_{j}$, of the primary ideals of $\mathfrak{B}_{j} A_{j+1}$, owing to the equality $\mathfrak{P}_{j}=\mathfrak{p} A_{j+1}$ and [7], Lemma 1.2,1), 2). This last property can be characterized through certain properties of $A_{j}$, as we show in the following lemma, which therefore gives a necessary condition for the property of $(*)$ we are studying. The following lemma is also a generalization of Lemma 1.3 of [7].

Lemma 2.1. Let $\mathfrak{p}$ be a maximal ideal of $A, A, A^{\prime}$ respectively be the ring obtained from $A$ by blowing-up $\mathfrak{p}$, and the gluing, over $\mathfrak{p}$, of the primary ideals belonging to $\mathfrak{p} \Lambda$. Then the following conditions are equivalent:

1) the rings $A, A^{\prime}$ coincide.

2) $\operatorname{emdim}\left(A_{p}\right)=e\left(A_{p}\right)$.

3) the conductor of $A$ in $A$ is p.

Proof. We put $S=A-\mathfrak{p}$, and we remember that $S^{-1} \Lambda$ is the ring obtained from $S^{-1} A$ by blowing-up $p S^{-1} A$. We have:

$1) \Rightarrow 2$ ) The gluing over $\mathfrak{p} S^{-1} A$ of the primary ideals of $\mathfrak{p} S^{-1} A$ is $B=S^{-1} A+\mathfrak{p} S^{-1} \Lambda([7]$, Lemma $1.2,1)$ ). Now, $\mathfrak{p} S^{-1} \Lambda \subset S^{-1} A$, since $\mathfrak{p} \Lambda \subset A^{\prime}$ ([7], Lemma 1.2, 1)) $\subset A$; then, $B \subset S^{-1} A$, so it is enough to apply [7], Lemma $1.3,1) \Rightarrow 2$ ).

2) $\Rightarrow 3$ ) Owing to [7], Lemma $1.3,2) \Rightarrow 3$ ), the conductor of $S^{-1} A$ in $S^{-1} A$ is $p S^{-1} A$. Let a be the conductor of $A$ in $A$; we have $\sqrt{a}=p$ (Lemma 1.1). Then, $\mathfrak{p} S^{-1} A=\mathfrak{a} S^{-1} A$, where $\mathfrak{a}$ is $p$-primary; it follows: $\mathfrak{p}=a$.

$3) \Rightarrow 1$ ) We have: $A^{\prime}=A+p A$ ([7], Lemma $\left.\left.1.2,1\right)\right) \subset A$, since $p$ is the conductor; so, $A^{\prime}=A$.

Owing to this lemma and the above remarks we have: the condition "emdim $\left(\left(A_{j}\right)_{\mathfrak{F}_{j}}\right)=e\left(\left(A_{j}\right)_{\mathfrak{B}_{j}}\right)$ for each $A_{j}$ in $(*)$ " is necessary to get the property of $(*)$ we are studying, but it is not sufficient (consider for example $A=k \llbracket t^{3}, t^{5}, t^{7} \rrbracket$ : the sequence $(*)$ is $A \subset k \llbracket t^{2}, t^{3} \rrbracket \subset k \llbracket t \rrbracket=\bar{A}$, where emdim $\left(\left(A_{j}\right)_{\Re_{j}}\right)=e\left(\left(A_{j}\right)_{\Re_{j}}\right)$ for each $A_{j}, \mathfrak{P}_{j}$, and (*) doesn't coincide with (**), as Proposition 3.2 of [7] shows). The following results allow us to find also sufficient conditions for the property of $(*)$ we are interested in.

The next lemma holds in the general case, not only for semilocal one-dimensional rings.

Lemma 2.2. Let $A \subset B$ be rings, $p$ a maximal ideal in $A, A^{\prime}$ be a ring between $A$ and $B$, such that $A^{\prime} \subset A+\mathfrak{p} B$. If $\mathfrak{p}^{\prime}$ is a prime ideal in 
$A^{\prime}$ over $\mathfrak{p}$ and $\mathfrak{p} B \neq B$, then $\mathfrak{p}^{\prime} B=\mathfrak{p} B$.

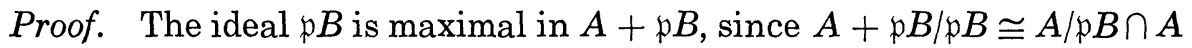
$=A / \mathfrak{p}$, which is a field. Besides, $\mathfrak{p} B=\left(\mathfrak{p} A^{\prime}\right) B \subset \mathfrak{p}^{\prime} B$, because $\mathfrak{p} A^{\prime} \subset \mathfrak{p}^{\prime}$; so, $\mathfrak{p} B \subset \mathfrak{p}^{\prime} B$, and $\mathfrak{p} B$ is maximal. It follows: $\mathfrak{p} B=\mathfrak{p}^{\prime} B$.

The next lemma recalls a well-known fact:

Lemma 2.3. Let $(A, \mathfrak{m}, k)$ be a local ring, $k=A / \mathfrak{m}$ and $M$ be a $k$ module. Then, $1_{A}(M)=1_{k}(M)$.

Proposition 2.4. Let $A, \mathfrak{p}, A$ be as in Lemma 2.1, $B$ be a ring between $\Lambda$ and $\bar{A}, \mathfrak{P}$ be a prime ideal in $B$ over $\mathfrak{p}$. Besides, let $\Lambda^{\prime}$ be the ring obtained from $B$ by blowing-up $\mathfrak{B}$. Let us suppose $B$ is a finite A-module, $\mathfrak{P}$ is the only prime ideal in $B$ over $\mathfrak{p}$, and the residue fields $k(\mathfrak{p}), k(\mathfrak{P})$ are canonically isomorphic. The following conditions are equivalent:

1) $\mathfrak{p} \Lambda^{\prime}=\mathfrak{P} \Lambda^{\prime}$

2) $e\left(A_{\mathfrak{p}}\right)=e\left(B_{\mathfrak{\beta}}\right)$.

Proof. We put: $R=A_{\mathfrak{q}}, S=B_{\Re}=B_{A-\downarrow}$ (see, for example, [1], p. 40), $L=\Lambda_{A-p}, L^{\prime}=\Lambda_{A-p}^{\prime}$. Then, $L$ is obtained from $R$ by blowing-up $\mathfrak{p} R$, so there is $x \in R, x$ regular in $L$ such that $\mathfrak{p} L=x L$ ([4], Proposition 1.1), and we have: $e(R)=1_{R}(R / x R)$ ([4], Remark a) p. 657) $=1_{R}\left(L^{\prime} \mid x L^{\prime}\right)$ ([4], Remark b) p. 657, where $J=L^{\prime}, x$ is regular in $R$ since is regular in $L$ ) $=1_{R}\left(L^{\prime} /(x L) L^{\prime}\right)=1_{R}\left(L^{\prime} /(p L) L^{\prime}\right)=1_{R}\left(L^{\prime} / p L^{\prime}\right)$. On the other hand, there is also $y \in B, y$ regular in $\Lambda^{\prime}$ and such that $\beta \Lambda^{\prime}=y \Lambda^{\prime}$ ([4] Proposition 1.1), so there is $y \in S, y$ regular in $L^{\prime}$, such that $\mathfrak{\beta} L^{\prime}=y L^{\prime}$. Then, as before we have: $e(S)=1_{S}\left(L^{\prime} / y L^{\prime}\right)=1_{S}\left(L^{\prime} / \Re L^{\prime}\right)$.

Besides, $L^{\prime} / \mathfrak{p} L^{\prime}$ (resp. $: L^{\prime} / \mathfrak{\beta} L^{\prime}$ ) is an $A / \mathfrak{p}=k(\mathfrak{p}$ )-module (resp. :a $B / \mathfrak{R}$ $=k(\Re)$-module), where the scalar product, induced by the structure of $L^{\prime}$, coincides with the inner product. Then, (Lemma 2.3) we have: $e(R)=$

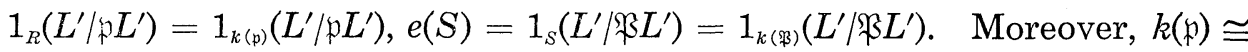
$k(\mathfrak{P})$. Then, if 1) holds, in particular $\mathfrak{p} L^{\prime}=\mathfrak{\beta} L^{\prime}$, so we have: $e(R)=$ $1_{h(p)}\left(L^{\prime} / p L^{\prime}\right)=1_{k(\mathfrak{p})}\left(L^{\prime} / \Re L^{\prime}\right)=1_{k(\mathfrak{\beta})}\left(L^{\prime} / \Re L^{\prime}\right)=e(S)$, i.e. 2). On the contrary, if 2) holds, $1_{k(\mathfrak{p})}\left(L^{\prime} / \mathfrak{p} L^{\prime}\right)=e(R)=e(S)=1_{k(\mathfrak{\beta})}\left(L^{\prime} / \Re L^{\prime}\right)=1_{k(\mathfrak{p})}\left(L^{\prime} / \Re L^{\prime}\right)$, so $M=$

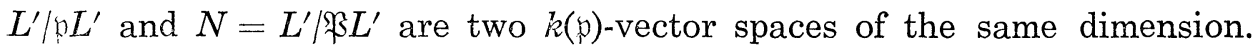
On the other hand, since $p L^{\prime} \subset \Re L^{\prime}$, we have: $M /\left(\Re L^{\prime} / p L^{\prime}\right)$ and $N$ are

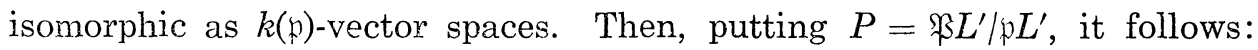
$\operatorname{dim}_{k(p)}(M)=\operatorname{dim}_{k(p)}(N)$, and also $\operatorname{dim}_{k(p)}(M)-\operatorname{dim}_{k(p)}(P)=\operatorname{dim}_{k(p)}(N)$. Therefore, $\operatorname{dim}_{k(p)}(P)=0$, so $\mathfrak{p} L^{\prime}=\mathfrak{P} L^{\prime}$; this equality implies $\mathfrak{p} \Lambda^{\prime}=\mathfrak{B} \Lambda^{\prime}$. 
From Proposition 2.4 and Lemma 2.2 it follows

Corollary 2.5. Let $A, B, A^{\prime}$ as in Proposition 2.4. If $B$ coincides with the gluing, over $\mathfrak{p}$, of the primary ideals belonging to $\mathfrak{p} \Lambda^{\prime}$, and $\mathfrak{P}$ is the only prime ideal of $B$ over $p$, the equivalent conditions of Proposition 2.4 are satisfied.

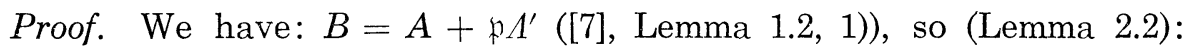
$\mathfrak{p} \Lambda^{\prime}=\mathfrak{s} \Lambda^{\prime}$, then 1) of Proposition 2.4 holds.

Using the above results and Section 1 we can find necessary and sufficient conditions in order that in $(*)$ each $A_{j}$ is a gluing, as required. We notice that in $(*)$ each blowing-up concerns a prime ideal $\mathbb{\beta}_{j} \in$ Ass $\left(A_{j} / \mathfrak{b}_{j}\right)$ such that $\mathfrak{P}_{j} \cap A \in \operatorname{Ass}(A / \mathfrak{b})$, where $\mathfrak{b}_{j}, \mathfrak{b}$ are respectively the conductor of $A_{j}$ in $\bar{A}$ and of $A$ in $\bar{A}$. In fact, according to the definition of (*), $\mathfrak{P}_{j}$ is an associated prime of the conductor a of $A_{j}$ in $A_{j+1}$ (Lemma 1.1); besides, $\mathfrak{b}_{j} \subset \mathfrak{a}$ since $A_{j} \subset A_{j+1} \subset \bar{A}$. Then, $\mathfrak{P}_{j} \supset \mathfrak{b}_{j}$, so $\mathfrak{P}_{j} \in \operatorname{Ass}\left(A_{j} / \mathfrak{G}_{j}\right)$. This implies: $\mathfrak{p}=\mathfrak{P}_{j} \cap A \in \operatorname{Ass}(A / \mathfrak{b})$. In fact, putting $S=A-\mathfrak{p}$, we have $S^{-1} A_{j} \subseteq S^{-1} \bar{A}$ (otherwise $\left(A_{j}\right)_{\Re_{j}}=\left(S^{-1} A_{j}\right)_{S^{-1} A_{j}-\Re_{j} S^{-1} A_{j}}=\left(S^{-1} \bar{A}\right)_{S^{-1} A_{j}-\mathfrak{F}_{j} S^{-1} A_{j}}=$ $\bar{A}_{A_{j}-\mathfrak{B}_{j}}$, with $\mathfrak{P}_{j} \in \operatorname{Ass}\left(A_{j} / \mathfrak{G}_{j}\right)$, contradiction); then, a fortiori we have: $S^{-1} A \subsetneq S^{-1} \bar{A}$, so $p \in \operatorname{Ass}(A / \mathfrak{b})$.

Let $\operatorname{Ass}(A / \mathfrak{b})=\left\{\mathfrak{h}_{1}, \cdots, \mathfrak{w}_{k}\right\}$. In general (see remark after Proposition 1.7), for each $\mathfrak{p}_{i} \in \operatorname{Ass}(A / \mathfrak{b})$ there are in $(*) n_{i} \geqslant 1$ rings obtained by blowing-up prime ideals which are over $p_{i}$. So, we write $(*)$ in such a way to point out this fact:

$$
\begin{gathered}
A=\Lambda_{1} \subset \cdots \subset \Lambda_{\jmath_{1}} \subset \Lambda_{\jmath_{1}+1} \subset \cdots \subset \Lambda_{\jmath_{2}} \subset \Lambda_{j_{2}+1} \\
\subset \cdots \subset \Lambda_{j_{k}} \subset \Lambda_{j_{k}+1}=\Lambda_{n}=\bar{A},
\end{gathered}
$$

meaning that, for $i=0, \cdots, k-1, A_{j_{i}+2}, \cdots, A_{j_{\imath+1}+1}$ are obtained by blowingup respectively $\mathfrak{P}_{j_{i}+1} \in \operatorname{Spec}\left(\Lambda_{j_{i}+1}\right), \cdots, \mathfrak{P}_{j_{i+1}} \in \operatorname{Spec}\left(\Lambda_{j_{i+1}}\right)$, where $\mathfrak{\beta}_{j_{i}+1} \cap A$ $=\cdots=\mathfrak{P}_{j_{i+1}} \cap A=\mathfrak{p}_{i+1}$ (we put: $j_{0}=0$ ).

Theorem 2.6. With the above notations, we assume: $k\left(\Re_{j}\right)=k(p)$ for each $\mathfrak{P}_{j} \in \operatorname{Spec} \Lambda_{j}, \mathfrak{p} \in \operatorname{Spec} A$ such that $\mathfrak{p}=\mathfrak{P}_{j} \cap A$. The following conditions are equivalent:

1) in the sequence $(*)^{\prime}$ each $\Lambda_{j}$ is the gluing, over $\mathfrak{p}=\mathfrak{R}_{j} \cap A$, of the primary ideals belonging to $\mathfrak{p} \Lambda_{j+1}(j=1, \cdots, n-1)$

2) for $j=1, \cdots, n-1, \mathfrak{P}_{j}$ is the only prime ideal in $A_{j}$ over $\mathfrak{p}=\mathfrak{x}_{j} \cap A$, and $\operatorname{emdim}\left(\left(\Lambda_{j}\right)_{\mathfrak{P}_{j}}\right)=e\left(\left(\Lambda_{j}\right)_{\mathfrak{F}_{j}}\right)=e\left(A_{p}\right)$. 
Proof. It is enough to prove: 1) $\Leftrightarrow 2$ ) for each $i=0, \cdots, k-1$ and each $j \in\left\{j_{i}+1, \cdots, j_{i+1}\right\}$.

Let us localize $(*)^{\prime}$ at $S=A-\mathfrak{p}_{i+1}$. We obtain:

$$
\begin{aligned}
A_{p_{i+1}}= & S^{-1} \Lambda_{1} \subset \cdots \subset S^{-1} \Lambda_{j_{i}} \subset S^{-1} \Lambda_{j_{i}+1} \subset S^{-1} \Lambda_{\jmath_{i}+2} \\
& \subset \cdots \subset S^{-1} \Lambda_{\jmath_{i+1}} \subset \cdots \subset S^{-1} \bar{A}
\end{aligned}
$$

where, for each $j, S^{-1} \Lambda_{j+1}$ is the ring obtained from $S^{-1} \Lambda_{j}$ by blowing-up $\mathfrak{P}_{j} S^{-1} \Lambda_{\jmath}$. Now, we have: $S^{-1} \Lambda_{2}=\cdots=S^{-1} \Lambda_{j_{i}+1}=A_{\mathfrak{p}_{i+1}}$. In fact, these rings are obtained by blowing-up prime ideals which are not over $\mathfrak{p}_{i+1} A_{\mathfrak{p}_{i+1}}$; so, after calling $a_{j}$ the conductor of $A_{p_{i+1}}$ in $S^{-1} \Lambda_{j}\left(j=2, \cdots, j_{i}+1\right)$, we have: $\sqrt{a_{j}}$ contains a product of prime ideals $\mathfrak{P}_{a_{1}} \ldots \mathfrak{P}_{a_{k}}$, where $\mathfrak{P}_{a_{1}} \cap A_{p_{i+1}} \neq$ $\mathfrak{p}_{i+1} A_{\mathfrak{p}_{i+1}}, \cdots, \mathfrak{P}_{a_{k}} \cap A_{\mathfrak{p}_{i+1}} \neq \mathfrak{p}_{i+1} A_{\mathfrak{p}_{i+1}}$ (see Lemma 1.1 and Lemma 1.4), so that no prime ideal belonging to $a_{j}$ coincides with $\mathfrak{p}_{i+1}$ for $j=2, \cdots, j_{i}+1$. Besides, $S^{-1} \Lambda_{j_{i+1}+1}=\cdots=S^{-1} \Lambda_{j_{k}}=S^{-1} \bar{A}=\overline{A_{p_{i+1}}}$. In fact, for $j=j_{i+1}+1$, $\cdots, j_{k}$, because of the definition of $(*)^{\prime}$, no prime ideal belonging to the conductor of $\Lambda_{j}$ in $\bar{A}$ lies over $\mathfrak{p}_{i+1}$, so that the conductor of $S^{-1} \Lambda_{j}$ in $S^{-1} \bar{A}=\overline{A_{p_{i+1}}}$ is not a proper ideal. Owing to these facts, the localization of $(*)^{\prime}$ at $S$ is:

$$
A_{p_{i+1}}=S^{-1} \Lambda_{j_{i}+1} \subset S^{-1} \Lambda_{j_{i}+2} \subset \cdots \subset S^{-1} \Lambda_{j_{i+1}} \subset \overline{A_{p_{i+1}}},
$$

where the first blowing-up concerns $\mathfrak{p}_{i+1} A_{\mathfrak{p}_{2+1}}$.

$1) \Rightarrow 2$ ) For each $j \in\left\{j_{i}+2, \cdots, j_{i+1}\right\}, \mathfrak{P}_{j}$ is the only prime ideal in $\Lambda_{\text {J }}$ over $\mathfrak{p}_{i+1}$ ([6], osserv. II); besides, $S^{-1} \Lambda_{j}$ contains the ring obtained from $A_{\mathfrak{p}_{i+1}}$ by blowing-up $\mathfrak{p}_{i+1} A_{\mathfrak{p}_{i+1}}$, it coincides with the gluing, over $\mathfrak{p}_{i+1} A_{p_{i+1}}$, of the primary ideals belonging to $\mathfrak{p}_{i+1} S^{-1} \Lambda_{j+1}$, and contains $\mathfrak{P}_{j} S^{-1} \Lambda_{j}$ as the only prime ideal over $\mathfrak{p}_{i+1} A_{\mathfrak{p}_{i+1}}$. Then (Corollary 2.5) we have: $e\left(A_{\mathfrak{p}_{i+1}}\right)=e\left(\left(S^{-1} \Lambda_{j}\right)_{\Re_{j} S^{-1} \Lambda_{j}}\right)$, so $e\left(A_{{p_{i}+1}}\right)=e\left(\left(\Lambda_{j}\right)_{\mathfrak{\Re}_{j}}\right)$ because $\left(S^{-1} \Lambda_{j}\right)_{\mathfrak{\beta}_{j} S^{-1} \Lambda_{j}}$ $=\left(\Lambda_{j}\right)_{\mathfrak{P}_{j}}$. Moreover, in $\Lambda_{j_{i}+1}, \mathfrak{P}_{j_{i}+1}$ is the only prime ideal over $\mathfrak{p}_{i+1}$, and we have also: $S^{-1} \Lambda_{j_{i}+1}=A_{\mathfrak{p}_{i+1}}$. So, $A_{\mathfrak{p}_{i+1}}=S^{-1} \Lambda_{j_{i}+1}=\left(\Lambda_{j_{i}+1}\right)_{\mathfrak{\beta}_{j_{i}+1}}$, then $e\left(A_{\mathfrak{p}_{i+1}}\right)=e\left(\left(\Lambda_{j_{i}+1}\right)_{\mathfrak{\beta}_{j_{i+1}}}\right)$. So, for $j \in\left\{j_{i}+1, \cdots, j_{i+1}\right\}$ we have: $e\left(A_{\mathfrak{p}_{i+1}}\right)=$ $e\left(\left(\Lambda_{j}\right)_{\mathfrak{x}_{j}}\right)$. On the other hand, $\Lambda_{j}$, being the gluing over $\mathfrak{p}_{i+1}$ of the primary ideals of $\mathfrak{p}_{i+1} \Lambda_{j+1}$, is also the gluing, over $\mathfrak{P}_{j}$, of the primary ideals of $\mathfrak{P}_{j} \Lambda_{j+1}$ ([7], Lemma 1.2,2)); then, owing to Lemma 2.1: emdim $\left(\left(\Lambda_{j}\right)_{\mathfrak{\Re}_{j}}\right)$ $=e\left(\left(\Lambda_{j}\right)_{\mathfrak{R}_{j}}\right)$. It follows: $\operatorname{emdim}\left(\left(\Lambda_{j}\right)_{\mathfrak{P}_{j}}\right)=e\left(\left(\Lambda_{j}\right)_{\mathfrak{F}_{j}}\right)=e\left(A_{\mathfrak{p}_{i+1}}\right)$ for $j \in\left\{j_{i}+1, \cdots\right.$, $\left.\dot{j}_{i+1}\right\}$.

2) $\Rightarrow 1)$ Let $i \in\{0, \cdots, k-1\}$. For each $j \in\left\{j_{i}+1, \cdots, j_{i+1}\right\}$, we have emdim $\left(\left(\Lambda_{j}\right)_{\mathfrak{R}_{j}}\right)=e\left(\left(\Lambda_{j}\right)_{\mathfrak{P}_{j}}\right)$, so (Lemma 2.1$): \Lambda_{j}$ coincides with the gluing, 
over $\mathfrak{P}_{\jmath}$, of the primary ideals of $\mathfrak{P}_{j} \Lambda_{j+1}$. Then, owing to [6], Proposition 1.5 we have: $\Lambda_{j}=\left\{x \in \Lambda_{j+1} \mid x \bmod \left(\mathfrak{P}_{j} \Lambda_{j+1}\right) \in f\left(k\left(\mathfrak{P}_{j}\right)\right)\right\}$, where $f$ is the canonical embedding: $k\left(\mathfrak{P}_{j}\right) \longrightarrow T^{-1}\left(\Lambda_{j+1} / \mathfrak{P}_{j} \Lambda_{j+1}\right), T=\Lambda_{j} / \mathfrak{P}_{j}-\{\overline{0}\}$. We want to prove: $\Lambda_{\jmath}$ is the gluing, over $\mathfrak{p}_{i+1}$, of the primary ideals of $\mathfrak{p}_{i+1} \Lambda_{\jmath+1}$, that is $\Lambda_{j}=\left\{x \in \Lambda_{j+1} \mid x \bmod \left(\mathfrak{p}_{i+1} \Lambda_{j+1}\right) \in \varphi\left(k\left(\mathfrak{p}_{i+1}\right)\right)\right\}$, where $\varphi$ is the canonical map: $k\left(\mathfrak{p}_{i+1}\right) \subset U^{-1}\left(\Lambda_{\jmath+1} / \mathfrak{p}_{i+1} \Lambda_{\jmath+1}\right), U=A / \mathfrak{p}_{i+1}-\{\overline{0}\}$.

Now, $U=k\left(\mathfrak{p}_{i+1}\right)-\{\overline{0}\}=k\left(\mathfrak{P}_{j}\right)-\{\overline{0}\}$ (for the assumptions) $=T$, so the hypothesis on $\Lambda_{j}$ can be written: $\Lambda_{j}=\left\{x \in \Lambda_{j+1} \mid x \bmod \left(\mathfrak{P}_{j} \Lambda_{j+1}\right) \in \varphi\left(k\left(\mathfrak{p}_{i+1}\right)\right)\right\}$, and it is enough to prove: $\mathfrak{p}_{i+1} \Lambda_{j+1}=\mathfrak{P}_{j} \Lambda_{j+1}$.

Let $S=A-\mathfrak{p}_{i+1}$. As before seen, for $j \in\left\{j_{i}+2, \cdots, j_{i+1}\right\}, S^{-1} \Lambda_{j}$ is local, with maximal ideal $\Re_{j} S^{-1} \Lambda_{j}$, and contains the ring $S^{-1} \Lambda_{\jmath_{i}+2}$, obtained from $A_{\mathfrak{p}_{i+1}}$ by blowing-up $\mathfrak{p}_{i+1} A_{\mathfrak{p}_{i+1}}$. Moreover, $e\left(\left(A_{j}\right)_{\mathfrak{\Re}_{j}}\right)=e\left(A_{\mathfrak{p}_{i+1}}\right)$, so $e\left(A_{\mathfrak{p}_{i+1}}\right)$ $=e\left(\left(S^{-1} \Lambda_{j}\right)_{\mathfrak{P}_{j} S^{-1} \Lambda_{j}}\right)$; besides, $k\left(\mathfrak{P}_{j} S^{-1} \Lambda_{j}\right)=k\left(\mathfrak{P}_{j}\right)=k\left(\mathfrak{p}_{i+1}\right)=k\left(\mathfrak{p}_{i+1} A_{\mathfrak{p}_{i+1}}\right)$. Then, owing to Proposition 2.4, we have: $\mathfrak{p}_{i+1} S^{-1} \Lambda_{j}=\mathfrak{P}_{j} S^{-1} \Lambda_{j}$, and this implies $\mathfrak{p}_{i+1} \Lambda_{j+1}=\mathfrak{P}_{\mathrm{j}} \Lambda_{j+1}$, for the assumptions on $S$. So, the result follows for $j \in\left\{j_{i}+2, \cdots, j_{i+1}\right\}$. As regards $\Lambda_{j_{i}+1}$, we know that $S^{-1} \Lambda_{j_{i}+1}=A_{p_{i+1}}$, so its maximal ideal $\mathfrak{P}_{j_{i}+1} S^{-1} \Lambda_{j_{i}+1}$ equals $\mathfrak{p}_{i+1} A_{p_{i+1}}=\mathfrak{p}_{\imath+1} S^{-1} \Lambda_{j_{l}+1}$; then, $\mathfrak{P}_{j_{i}+1} S^{-1} \Lambda_{j_{i}+2}=\mathfrak{p}_{i+1} S^{-1} \Lambda_{j_{i}+2}$. Then, the result follows for each $j \in$ $\left\{j_{i}+1, \cdots, j_{i+1}\right\}$.

Now, we show certain classes of rings, such that $(*)$ satisfies the two equivalent conditions of Theorem 2.6.

Corollary 2.7. Under the same assumptions as in Theorem 2.6, a ring $A$ such that ${ }^{A} \sqrt{\mathfrak{b}}=\mathfrak{b}, \mathfrak{b}=A:{ }_{A} \bar{A}$, satisfies condition 1) of Theorem 2.6.

Proof. We shall prove that $A$ satisfies 2) of Theorem 2.6; it is enough to show that this condition holds for each $i \in\{0, \cdots, k-1\}, j \in\left\{j_{i}+1, \cdots\right.$, $\left.j_{i+1}\right\}$, if $\sqrt[A]{\mathfrak{b}}=\mathfrak{b}$. So, let $i \in\{0, \cdots, k-1\}, S=A-\mathfrak{p}_{i+1}$. At the beginning of the proof of Theorem 2.6 we showed that the localization of $(*)^{\prime}$ at $S$ is:

$$
A_{p_{i+1}}=S^{-1} \Lambda_{j_{i}+1} \subset S^{-1} \Lambda_{j_{i}+2} \subset \cdots \subset S^{-1} \Lambda_{\jmath_{i}+1} \subset \overline{A_{p_{i+1}}^{--}} .
$$

In this particular case, we have: $A_{p_{i+1}}=S^{-1} \Lambda_{j_{i}+1} \subset S^{-1} \Lambda_{j_{i}+2}=\cdots=A_{p_{i+1}}$, since (as we shall prove) the conductor of $S^{-1} \Lambda_{j_{i}+2}$ in $\overline{A_{p_{i+1}}}$ is not a proper ideal. Let $\mathfrak{b}_{j_{i}+2}$ be the conductor of $\Lambda_{j_{i}+2}$ in $\bar{A}$; then, the conductor of $S^{-1} \Lambda_{j_{i}+2}$ in $\overline{A_{\mathfrak{p}_{i+1}}}$ is $\mathfrak{b}_{j_{i}+2} S^{-1} \Lambda_{j_{i}+2}$. If this ideal is proper, it is the intersection of the prime ideals $\mathfrak{P}_{a_{1}}, \cdots, \mathfrak{P}_{a_{r}}$ of $S^{-1} \Lambda_{j_{i}+2}$ such that $\left\{\mathfrak{P}_{a_{j}} \cap A_{p_{i+1}}\right.$, $j=0, \cdots, r\}=\operatorname{Ass}\left(A_{p_{i+1}} / \mathfrak{b} A_{p_{i+1}}\right)-\left\{\mathfrak{p}_{i+1} A_{p_{i+1}}\right\}$ (see Corollary 1.10 ); but $\mathfrak{b} A_{p_{i+1}}$ $=\mathfrak{f}_{i+1} A_{\mathfrak{p}_{i+1}}$, since $\mathfrak{b}=\sqrt[A]{\mathfrak{b}}$, so $\mathfrak{b}_{j_{i}+2} S^{-1} \Lambda_{j_{i}+2}$ is not proper. 
So, it follows that in $(*)^{\prime}$ the only "link" concerning blowing-up of prime ideals over $\mathfrak{p}_{i+1}$ is $\Lambda_{j_{i}+1} \subset \Lambda_{j_{2}+2}$; then, it is enough to show that $\Lambda_{j_{i}+1}$ satisfies 2) of Theorem 2.6. Indeed, we have: in $\Lambda_{j_{i}+1}, \mathfrak{P}_{j_{i+1}}$ is the only prime ideal over $\mathfrak{p}_{i+1}$, because $S^{-1} \Lambda_{j_{\imath}+1}=A_{p_{i+1}}$ is local, and its maximal ideal is $\mathfrak{P}_{j_{i}+1} S^{-1} \Lambda_{j_{i}+1}$. So, we have also: $\left(\Lambda_{j_{i}+1}\right)_{\mathfrak{P}_{j_{i}+1}}=S^{-1} \Lambda_{j_{i}+1}([1]$, p. 40$)=A_{\mathfrak{p}_{i+1}}$. Besides, since $\sqrt[A]{\sqrt[b]{\mathfrak{b}}}=\mathfrak{b}$, the conductor of $A_{\mathfrak{p}_{i+1}}$ in $\overline{A_{\mathfrak{p}_{i+1}}}$ is $\mathfrak{p}_{i+1} A_{\mathfrak{p}_{i+1}}$, then, owing to the above facts, we have also: the conductor of $S^{-1} \Lambda_{j_{i}+1}$ in $S^{-1} \Lambda_{j_{i}+2}$ is $\mathfrak{p}_{i+1} S^{-1} \Lambda_{j_{i}+1}$, which equals $\mathfrak{P}_{j_{i}+1} S^{-1} \Lambda_{j_{i}+1}$. It follows (Lemma 2.1): $\operatorname{emdim}\left(\left(S^{-1} \Lambda_{j_{i}+1}\right)_{\mathfrak{P}_{j_{i}+1} S^{-1} \Lambda_{j_{2}+1}}\right)=e\left(\left(S^{-1} \Lambda_{j_{i}+1}\right)_{\mathfrak{F}_{j_{i}+1} S^{-1} \Lambda_{j_{i}+1}}\right)$, i.e. $\operatorname{emdim}\left(\left(\Lambda_{j_{i}+1}\right)_{\mathfrak{P}_{j_{i}+1}}\right)$ $=e\left(\left(\Lambda_{j_{i}+1}\right)_{\mathfrak{F}_{j_{i}+1}}\right)$. So, $\Lambda_{j_{i}+1}$ is as required.

Corollary 2.8. Under the same assumptions as in Theorem 2.6, if $A$ is seminormal, then A satisfies condition 1) of Theorem 2.6.

Proof. If $A$ is seminormal, then $\sqrt[A]{\sqrt[b]{b}}=\mathfrak{b}$; so, we can apply Corollary 2.7 .

Corollary 2.9. Under the same assumptions as in Theorem 2.6, let $A$ be local, analytically irreducible and such that emdim $(A)=2$. Then, condition 1) of Theorem 2.6 holds if and only if $e(A)=2$.

Proof. If $A$ satisfies 1) of Theorem 2.6, in particular we have: $e(A)$ $=\operatorname{emdim}(A)=2$. (Theorem 2.6). On the contrary, suppose $e(A)=2$. For each $\Lambda_{j}$ in $(*)^{\prime}, \Lambda_{j}$ is a local ring (since $\bar{A}$ is a discrete valuation ring, see [3], p. 748), so it is enough to prove: emdim $\left(\Lambda_{j}\right)=e\left(\Lambda_{j}\right)=e(A)=2$. Let $\mathfrak{m}$ (resp.: $\mathfrak{B}_{j}$ ) be the maximal ideal of $A$ (resp.: of $\Lambda_{j}$ ). We have: $e\left(\Lambda_{j}\right) \leqslant e(A)$. In fact, $e(A)=1_{A}(A / x A)$ (for a suitable regular $\left.x\right)=1_{A}(\bar{A} / \mathrm{m} \bar{A})$ (see [4], Remark a), b) p. 657, Lemma 1.8), and also $e\left(\Lambda_{j}\right)=1_{\Lambda_{j}}\left(\bar{\Lambda}_{j} / \Re_{j} \bar{\Lambda}_{j}\right)$ (see [4], as above). Now, $\bar{\Lambda}_{j}=\bar{A}$, so $e\left(\Lambda_{j}\right)=1_{\Lambda_{j}}\left(\bar{A} / \Re_{j} \bar{A}\right)$. Besides, owing to Lemma 2.3, putting $k=k(\mathfrak{m})=k\left(\Re_{j}\right)$, we have: $1_{A}(\bar{A} / \mathfrak{m} \bar{A})=1_{k}(\bar{A} / \mathfrak{n} \bar{A})$, $1_{\Lambda_{j}}\left(\bar{A} / \Re_{j} \bar{A}\right)=1_{k}\left(\bar{A} / \Re_{j} \bar{A}\right)$. We have also: $\bar{A} / \Re_{j} \bar{A}$ is isomorphic to $(\bar{A} / \mathrm{m} \bar{A}) /\left(\mathfrak{B}_{j} \bar{A} / \mathrm{m} \bar{A}\right)$ as a $k$-vector space. So, $e\left(\Lambda_{j}\right)=1_{k}\left(\bar{A} / \Re_{j} \bar{A}\right)=1_{k}(\bar{A} / \mathfrak{m} \bar{A})$ $-1_{k}\left(\Re_{j} \bar{A} / m \bar{A}\right) \leqslant 1_{k}(\bar{A} / m \bar{A})=e(A)$.

Then, we have: emdim $\left(\Lambda_{j}\right) \leqslant e\left(\Lambda_{j}\right)([4]$, Corollary 1.10$) \leqslant e(A)$ (as before seen $)=2$. On the other hand, emdim $\left(\Lambda_{j}\right) \geqslant 2$, because $\Lambda_{j}$ is not regular. It follows: emdim $\left(\Lambda_{j}\right)=e\left(\Lambda_{j}\right)=e(A)=2$.

So, Corollary 2.9 shows that, if $C$ is an analytically irreducible plane curve with singular point $P$, the local ring of $C$ at $P$ satisfies condition 1) of Theorem 2.6 if and only if $P$ is a double point. Also for a larger 
class of analytically irreducible curves we can characterize the rings $A$ satisfying condition 1) of Theorem 2.6: see the next Corollary 2.10, which shows how Proposition 2.3 of [7] can be deduced from Theorem 2.6.

Let $A$ be the local ring of a monomial curve: $A=k \llbracket t^{n_{1}}, \cdots, t^{n_{p}} \rrbracket$, with $k$ algebrically closed. By $S=\left\langle n_{1}, \cdots, n_{p}\right\rangle$ we denote the semigroup generated by $n_{1}, \cdots, n_{p}$.

Corollary 2.10. Let $A=k \llbracket t^{n_{1}}, \cdots, t^{n_{p}} \rrbracket$, where $n_{1}<\cdots<n_{p}$ generate minimally $S=\left\langle n_{1}, \cdots, n_{p}\right\rangle$. Then, condition 1) of Theorem 2.6 holds if and only if $n_{2} \equiv 1 \bmod \left(n_{1}\right), n_{j}=n_{j-1}+1$ for $3 \leqslant j \leqslant p$.

Proof. Since each $\Lambda_{j}$ in $(*)$ is local, it is enough to prove: "emdim $\left(\Lambda_{j}\right)$ $=e\left(\Lambda_{j}\right)=e(A)$ for $j=1, \cdots, n-1$ " if and only if “ $n_{2} \equiv 1 \bmod \left(n_{1}\right), n_{h}=$ $n_{h-1}+1$ for $3 \leqslant h \leqslant p$ " (see Theorem 2.6). One has: the condition " $e\left(\Lambda_{j}\right)$ $=e(A)$ for $j=1, \cdots, n-1$ " is equivalent to " $n_{2} \equiv 1 \bmod \left(n_{1}\right)$ ". In fact, if $e\left(\Lambda_{j}\right)=e(A), j \in\{1, \cdots, n-1\}$ then $e\left(\Lambda_{j}\right)=n_{1}$; it implies that the remainder $r$ of the division of $n_{2}$ by $n_{1}$ is equal to 1 , otherwise there is a $\Lambda_{j}$ such that $e\left(\Lambda_{j}\right)=r<n_{1}$, for a $j \in\{1, \cdots, n-1\}$ (see [7], Lemma 2.1). Contrariwise, if $n_{2} \equiv 1 \bmod \left(n_{1}\right)$, owing to Lemma 2.1 of [7] we have: $e\left(\Lambda_{j}\right)=n_{1}$ for $j=1, \cdots, n-1$, so $e\left(\Lambda_{j}\right)=e(A)$

Now, it is enough to apply Proposition 3.1 and Theorem 1.5 of [7], to complete the proof.

\section{REFERENCES}

[1] N. Bourbaki, Algebre Commutative Ch. 5-6, Hermann, Paris, 1964.

[2] S. Greco and P. Valabrega, On the theory of adjoints, Lecture Notes in Math., 732 (Algebraic Geometry) Springer-Verlag (1978), 98-123.

[3] E. Kunz, The value-semigroup of a one-dimensional Gorenstein ring, Proc. of Amer. Math. Soc., 25 (1970), 748-751.

[4] J. Lipman, Stable ideals and Arf rings, Amer. J. Math., 93 (1971), 649-685.

[ 5 ] E. Matlis, 1-Dimensional Cohen-Macaulay rings, Lecture Notes in Math., 327 Springer-Verlag (1970).

[6 ] G. Tamone, Sugli incollamenti di ideali primari e la genesi di certe singolarità, B.U.M.I. (Supplemento) Algebra e Geometria, Suppl., 2 (1980), 243-258.

[ 7] - Blowing-up and gluings in one-dimensional rings to appear on Commutative Algebra: Proceedings of the Trento Conference, Lecture Notes in pure and applied Math., M. Dekker Inc.

Istituto di Matematica

Università di Genova

Via L. B. Alberti \&

16132 Genova

Italy 